\title{
Mamíferos não-voadores e ataques por cães domésticos em Morro Branco, Vargem Alta, ES
}

A redução populacional de mamíferos é causada principalmente pela perda e fragmentação de habitats, em especial, no contexto da Mata Atlântica, além da caça e a introdução de espécies exóticas em ambientes naturais, como os cães domésticos. Este estudo teve como objetivo realizar um levantamento de mamíferos não-voadores e avaliar o impacto predatório de Canis ( Foram registrados 18 táxons, distribuídos em 6 ordens e 10 familias, totalizando 84 indivíduos. Três táxons foram classificados como abundantes (CTA): Nasua nasua (26\%), Canis lupus familiaris (15\%) e Didelphis aurita (14\%). Quatro espécies constam como ameaçadas: Puma yagouaroundi, Puma concolor, Mazama Bororo e Callithrix flaviceps. A técnica que registrou maio número de táxons foi o registro de carcaças de animais predados, com 9 táxons, seguido de registro visual, com 8 espécies e uso de armadilha fotográfica, com 7 espécies. 0 registro de carcaças de animais predados por Canis lupus familiaris foi o método com maior número de registros exclusivos, como Puma yagouaroundi, Galisctis cuja e Mazama bororo, registrados apenas desta forma. 13 animais foram encontrados abatidos, com marcas de mordidas evidentes, correspondendo a $18 \%$ do total. As espécies mais predadas foram Didelphis aurita, Dasypus novemcinctus e Mazama americana. Canis lupus familiaris apresentou alta Abundância Relativa $(15,48 \%)$ na área, tornando-se um fator de risco para a conservação de espécies ameaçadas, como Puma yagouaroundi e Puma concolor, que além de ataques, podem sofrer por competição. É urgente a tomada de ações conservacionistas e a elaboração de um plano de manejo para a criação da Área de Proteção Ambiental (APA) 'Morro Branco', tendo em vista a influência de animais exóticos sobre a fauna nativa, implicando diretamente na conservação da biodiversidade.

Palavras-chave: Mamíferos; Canis lupus familiaris; Mata Atlântica; Conservação; Espécies exóticas invasoras.

\section{Non-flying mammals and attacks by domestic dogs in Morro Branco, Vargem Alta, ES}

\begin{abstract}
The reduction in the population of mammals is caused mostly by the loss and habitat fragmentation, especially in the context of the Atlantic Forest, in addition to hunting and the introduction of exotic species in natural environments, as the domestic dogs. The objective of this study was to accomplish a survey of non-flying mammals and to evaluate the predatory impact of Canis lupus familiaris on this community, in an area selected for the implementation of the future Environmental Protection Area (APAs) 'Morro Branco', at Sítio Morro Branco, Vargem Alta, Espírito Santo State, from february of 2015 to february of 2016. The survey orcurred by active search for traces (footprints, feces, and carcasses), and using cage traps and camera traps. Eighteen Santo State, from february of 2015 to february of 2016 . The survey occurred by active search for traces (footprints, feces, and carcasses), and using cage traps and camera traps. Eighteen
taxa were registered, distributed in 6 orders and 10 families, totaling 84 individuals. Three taxa were classified as abundant (CTA): Nasua nasua (26\%), Canis lupus familiaris (15\%), and taxa were registered, distributed in 6 orders and 10 families, totaling 84 individuals. Three taxa were classified as abundant (CTA): Nasua nasua (26\%), Canis lupus familiaris (15\%), and
Didelphis aurita (14\%). Four species are threatened with extinction: Puma yagouaroundi, Puma concolor, Mazama Bororo, and Callithrix flaviceps. The technique that registered the highest Didelphis aurita (14\%). Four species are threatened with extinction: Puma yagouaroundi, Puma concolor, Mazama Bororo, and Callithrix flaviceps. The technique that registered the highest
number of taxa was the record of carcasses of predated animals with 9 species, followed by the visual record with 8 species, and the use of camera trap, registering 7 species. The registration number of taxa was the record of carcasses of predated animals with 9 species, followed by the visual record with 8 species, and the use of camera trap, registering 7 species. The registration registered only in this way. Third by Canis lupus familiaris was the with evident bite marks, corresponding to $18 \%$ of the total. The most predated species were Didelphis aurita, Dasypus novemcinctus, and Mazama Americana. Canis lupus familiaris presented a high Relative Abundance $15,48 \%$ in the area, becoming a risk factor for the conservation of endangered species as Puma yasouroundi, and Puma conclor, which besides attacks, can suffer by competition. It is urgent to take conservationist actions, and to elaborate a management plan for the creation of the Environmental Protection Area (APAs) 'Morro Branco', in view of the effect of exotic animals on native fauna, affecting the conservation of biodiversity.
\end{abstract}

Keywords: Mammals; Canis lupus familiaris; Atlantic forest; Conservation; Invasive alien species.

Topic: Conservação da Biodiversidade

Reviewed anonymously in the process of blind peer.
Received: 05/12/2020

Approved: 20/02/2021
Helimar Rabello (iD

Centro Universitário São Camilo, Brasil http://lattes.cnpq.br/3789680363736207 http://orcid.org/0000-0002-1699-595X helimarbio@hotmail.com

Luan Gonçalves Bissa (iD

Centro Universitário São Camilo, Brasi http://lattes.cnpq.br/1550232803551374 http://orcid.org/0000-0001-7262-0805 luan.bio@hotmail.com

Gabriel Permanhane da Silva (iD) Universidade Federal do Espírito Santo, Brasil http://lattes.cnpq.br/4064111590440315 http://orcid.org/0000-0002-9546-2341 permanhaneg@gmail.com
Gilson Silva Filho (iD)

Centro Universitário São Camilo, Brasil http://lattes.cnpq.br/1040363193594196 http://orcid.org/0000-0001-5313-1424 silva.filho.gilson@gmail.com

Cíntia Cristina Lima Teixeira

Centro Universitário São Camilo, Brasil http://lattes.cnpq.br/2013098051038491 http://orcid.org/0000-0002-8438-5897 cintiateixeira@saocamilo-es.br

Tatiana Gorone Fávero Pin (DD

Centro Universitário São Camilo, Brasil http://lattes.cnpq.br/8983540149349160 http://orcid.org/0000-0001-5180-2968 tatianagorone@gmail.com
Leticia Rigo Tavares (iD)

Universidade Federal do Espírito Santo, Brasil http://lattes.cnpq.br/7338379941864880 http://orcid.org/0000-0002-3992-4185 leticiarigot@hotmail.com

DOI: 10.6008/CBPC2318-2881.2021.001.0005

Referencing this:

RABELLO, H.; BISSA, L. G.; SILVA, G. P.; SILVA FILHO, G.; TEIXEIRA, C. C. L.; PIN, T. G. F.; TAVARES, L. R.. Mamíferos não-voadores e ataques por cães domésticos em Morro Branco, Vargem Alta, ES. Nature and Conservation, v.14, n.1, p.41-54, 2021. DOI: http://doi.org/10.6008/CBPC2318-2881.2021.001.0005 


\section{INTRODUÇÃO}

A Mata Atlântica é um dos biomas mais afetados pelo desmatamento e fragmentação, com forte redução em sua extensão (FERNANDEZ, 1997; TABARELLI et al., 2005; RIBEIRO et al., 2009), que originalmente compreendia cerca de $16 \%$ do território nacional. Atualmente, se encontra reduzida a 8,5\% do seu tamanho original e, no Estado do Espírito Santo, restam apenas $11,2 \%$ do território cobertos por florestas desse bioma, sendo em sua maioria representados por Unidades de Conservação (UCs) (IPEMA, 2011).

Neste contexto de fragmentação, a Mata Atlântica ainda abriga uma elevada diversidade de mamíferos, sendo o segundo hotspot em termos de diversidade da classe, com cerca de 298 espécies (PAGLIA et al., 2012). No Espírito Santo, a Mata Atlântica também detém altíssima riqueza biológica de mamíferos (MENDES, 1995; CHIARELLO, 1999; PASSAMANI et al., 2000; HELDER-JOSÉ et al., 2016). Em relação ao número de espécies ocorrentes no Espírito Santo, a família Chiroptera (54) é a mais abundante, seguida de Rodentia (34), Didelphidae (13), Xenarthra (9), Primates (7) e Felidae (6) (MOREIRA et al., 2008).

Os mamíferos podem atuar como indicadores ecológicos, refletindo o estado de conservação do ecossistema em que estão inseridos (MENEZES et al., 2013). A perda e a fragmentação de habitats, seguidos da caça e da introdução de espécies exóticas, afetam diretamente a mastofauna (BAILLIE et al., 2004; GALETTI et al., 2009). Segundo Bierregaard et al. (1992), essas alterações no ambiente modificam sua abundância e diversidade.

A interação com cães domésticos é um fator relevante na perda de mamíferos silvestres (YOUNG et al, 2011; HUGHES et al., 2013), à medida em que matam, perseguem, perturbam, causam deslocamento de fauna nativa (RANGEL et al., 2013) e hibridam com espécies de canídeos selvagens (HUGHES et al., 2013). Canis lupus familiaris apresenta comportamento de fauna exótica invasora quando deixa a vida doméstica e passa a pertencer ao ambiente de forma selvagem (RANGEL et al., 2013). Possuem tendência a caçar por diversão, presas grandes ou pequenas, raramente consumindo suas vítimas (GREEN et al., 1994; VEITCH, 2002; BRICKNER, 2003; ESPARTOSA, 2009).

Espartosa (2009) observa que é comum o avistamento de cães em áreas de floresta nativa, mesmo quilômetros mata à dentro, estando presentes em maior densidade que a maioria dos mamíferos nativos. Apesar deste cenário, ainda são recentes e pouco numerosos os estudos sobre o impacto da predação de cães domésticos sobre animais selvagens, principalmente no Brasil (GALETTI et al., 2006; CAMPOS et al., 2007; OLIVEIRA et al., 2008; SRBEK-ARAUJO et al., 2008; HUGHES et al., 2013; RANGEL et al., 2013; PEREIRA et al., 2019).

Para se explicar o efeito dos cães sobre a comunidade de mamíferos, é preciso conhecer a distribuição e abundância das espécies (AHUMADA et al., 2011; GALETTI et al., 2015). Ademais, estudos sobre a mastofauna são de grande importância, principalmente em região de Mata Atlântica, dada sua alta diversidade e endemismos (BROOKS et al., 2002; CEBALLOS et al., 2006), fornecendo subsídios para avaliar o estado de conservação das espécies (IWANAGA, 2004) e possibilitando o desenvolvimento de planos de conservação e manejo (PARDINI et al., 2006). 
O presente estudo teve como objetivo realizar um levantamento de mamíferos não-voadores e avaliar o impacto de cães (Canis lupus familiaris) sobre esta comunidade, em uma área selecionada para a implantação da futura Área de Proteção Ambiental (APA) “Morro Branco". Os resultados poderão auxiliar no desenvolvimento do futuro plano de manejo da unidade, além de contribuir com novos dados referentes à mastofauna da região sul-capixaba.

\section{METODOLOGIA}

O sítio Morro Branco está localizado na rodovia ES-164 no sentido Vargem Alta - Vila Esperança, distante apenas três quilômetros do centro, nas coordenadas (24K-291050.63 UTM 77.1460). Trata-se de uma propriedade com área total de $37.700 \mathrm{~m}^{2}$ (Figura 1).

A área é ocupada por plantios de Eucalyptus e Pinus sp., possui aproximadamente um terço de sua área coberta por mata nativa com a tipologia florestal de Mata Atlântica Ombrófila Submontana, em estágio secundário de regeneração e dois hectares compreendendo uma área de lazer. Uma parte da área de Pinus $s p$. ., equivalente a $3.000,00 \mathrm{~m}^{2}$, está sendo derrubada para a efetivação de pomar tipo SAF (Sistema Agroflorestal), o que beneficiará a fauna local. A Figura 1 a seguir se refere aos limites da área em amarelo e os três transectos em vermelho (T1:24k290792.12/7714792.68; T2:24k290879.68/UTM 7715230.47; T3: 24k291195.02/ 7715333.46) onde as armadilhas foram alocadas.

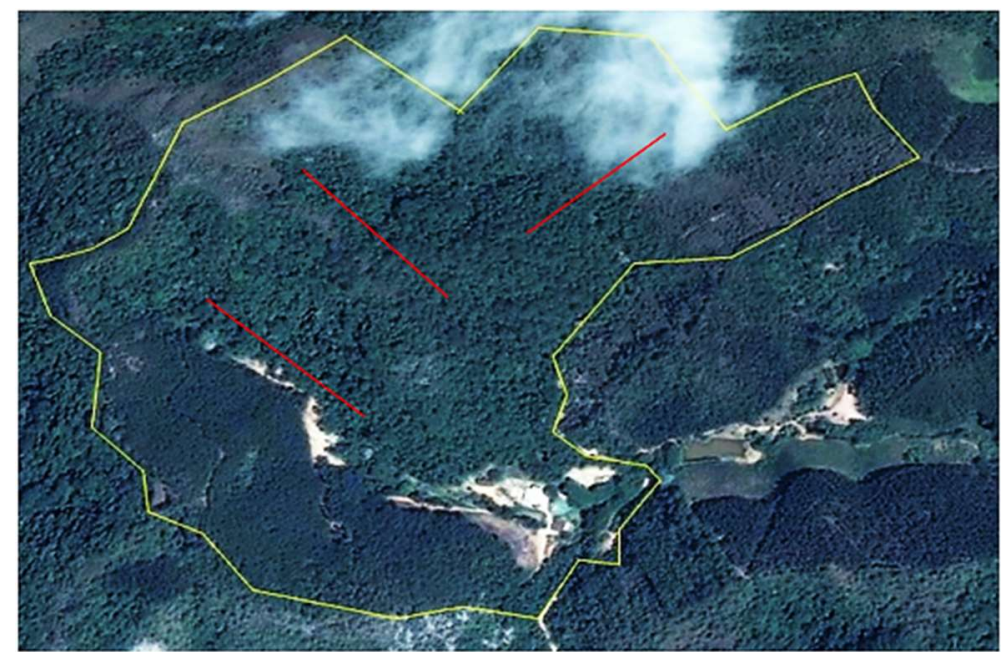

Figura 1: Carta imagem com a área da propriedade Sítio Morro Branco, Vargem Alta, ES. Fonte: Google Earth.

A coleta de dados foi realizada numa média de 2 excursões mensais entre os meses de fevereiro de 2015 e fevereiro de 2016. As armadilhas eram alocadas na sexta à noite por volta das 18 horas e retiradas no domingo, às 18 horas, totalizando um período de 12 meses e um esforço amostral de 1.104 horas de campo. A metodologia se baseou em busca ativa por vestígios e uso de armadilhas fotográficas.

Os equipamentos utilizados foram: 6 armadilhas fotográficas/filmadoras Bushnell ZT820; 18 armadilhas de gaiola Tomahawk tamanho $515 \times 480$ e $651 \times 606$, dimensão para animais de pequeno e médio porte; iscas alimentares para atrativo de fauna; Câmeras Digitais Canon SX10ls e Sony H50; GPS Garmin Rino 110, para o georreferenciamento dos pontos analisados; computador de mão Acer Aspire 5538 para registro de imagens capturadas; fitas métricas; lápis; e caderneta de campo. 
Para alocação das gaiolas e armadilhas fotográficas, foram efetuados três transectos, em área de vegetação em estágio secundário de regeneração, todos eles em trilha de mata, sendo dois deles margeados por um córrego. As armadilhas foram iscadas diariamente durante as excursões, utilizando-se uma mistura de óleo de fígado de bacalhau, farinha de milho e banana. Também foram utilizados como iscas frutas, carne bovina, miúdos de frango e sardinha. As mesmas eram verificadas a cada 3 horas. As gaiolas foram posicionadas a uma distância de 30 metros uma da outra e os indivíduos capturados foram fotografados, identificados em campo, quando possível, e posteriormente liberados.

A busca ativa foi efetuada percorrendo duas estradas que atravessam os fragmentos de mata e os plantios florestais, perfazendo um trajeto de $5 \mathrm{~km}$, ao amanhecer e ao entardecer, fotografando todos os animais e vestígios como tocas, pegadas e carcaças. As carcaças de animais encontradas eram fotografadas e algumas, em melhor estado, coletadas e enviadas ao Laboratório de Zoologia do Centro Universitário São Camilo - ES, para posterior taxidermização. Para confecção dos moldes de pegadas foram utilizados 9 moldes de PVC, com 3 tamanhos diferenciados, de 150, 100 e 50 polegadas e gesso de secagem rápida.

Para identificação das espécies utilizou-se literatura específica (PAZ et al., 2003; REIS et al., 2011), bem como para a identificação dos vestígios (BECKER et al., 1991). A curva do coletor, os estimadores de riqueza e diversidade foram realizados com os softwares PAST e EstimateS. As Frequências de Ocorrência (FO) e Abundância Relativa (AR) foram obtidas, para cada táxon, através das seguintes equações:

$(\mathrm{FO})=($ № de dias com registro da espécie/ № de campanhas) * 100

$(A R)=($ Abundância da espécie/ Abundância Total de Mastofauna) $* 100$

\section{RESULTADOS E DISCUSSÃO}

Registrou-se, ao todo, 84 mamíferos não-voadores, pertencentes à 18 espécies, distribuídas em 6 ordens e 10 famílias (Tabela 1). A ordem com o maior número de espécies foi Carnívora ( $n=7$ ), correspondendo à 39\%, seguida de Didelphimorphia $(n=4)$, correspondendo a $22 \%$, Cingulata $(n=2)$, Rodentia $(n=2)$ e Artiodactyla $(n=2)$, representando $11 \%$ cada e Primates $(n=1)$, correspondendo a apenas $5 \%$ dos táxons.

A ordem com maior representatividade em famílias também foi Carnívora, com 4 famílias, seguido de Rodentia, com 2 famílias, enquanto Cingulata, Didelphimorphia, Primates e Artiodactyla apresentaram apenas uma família cada. Em relação à representatividade por família, Didelphidae $(n=4)$ apresentou o maior número de táxons, seguido de Felidae $(n=3)$, Dasypodidae, Mustelidae e Cervidae que apresentaram duas espécies cada ( $n=2)$, enquanto Callitrichidae, Procyonidae, Canidae, Sciuridae e Cuniculidae apresentaram uma espécie cada $(n=1)$.

Com exceção de Canis lupus familiaris, que é uma espécie exótica, e de Guerlinguetus ingrami, todas as outras espécies aparecem no último Livro Vermelho da Fauna Brasileira (ICMBIO, 2018). Dessas, quatro são consideradas ameaçadas: Puma yagouaroundi, Puma concolor e Mazama bororo, categorizadas como Vulnerável (VU) e Callithrix flaviceps, como Em Perigo (EN). Na lista das espécies do Espírito Santo Ameaçadas de Extinção (IEMA, 2005), Callithrix flaviceps e Puma concolor aparecem Em Perigo (EM), e Leopardus 
pardalis, como Vulnerável (VU).

Tabela 1: Espécies de mamíferos não-voadores registrados no Sítio Morro Branco, Vargem Alta, ES. Ordem, Família, Espécie, Nome popular e ameaças. BR= Livro Vermelho da Fauna Brasileira (ICMBIO, 2018); ES= Lista da fauna ameaçada de extinção no Espírito Santo (IEMA, 2005). VU= Vulnerável; EM= Em perigo.

\begin{tabular}{|c|c|c|c|c|}
\hline \multirow{2}{*}{$\begin{array}{l}\text { Ordem / Família } \\
\text { ARTIODACTYLA }\end{array}$} & \multirow[t]{2}{*}{ Espécie } & \multirow[t]{2}{*}{ Nome popular } & \multicolumn{2}{|c|}{ Ameaças } \\
\hline & & & BR & ES \\
\hline \multirow[t]{2}{*}{ Cervidae } & Mazama americana (Erxleben, 1777) & Veado-mateiro & & \\
\hline & Mazama bororo Duarte, 1996 & Veado-mateiro-pequeno & VU & \\
\hline \multicolumn{5}{|l|}{ CARNIVORA } \\
\hline Canidae & Canis lupus familiaris (Linnaeus, 1758) & Cão doméstico & & \\
\hline \multirow[t]{3}{*}{ Felidae } & Leopardus pardalis (Linnaeus, 1758) & Jaguatirica & & VU \\
\hline & Puma concolor (Linnaeus, 1771) & Onça Parda & VU & EM \\
\hline & Puma yagouaroundi (É. Geoffroy, 1803) & Gato Mourisco & VU & \\
\hline \multirow[t]{2}{*}{ Mustelidae } & Eira barbara (Linnaeus, 1758) & Irara & & \\
\hline & Galictis cuja (Molina, 1782) & Furão & & \\
\hline Procyonidae & Nasua nasua (Linnaeus, 1766) & Quati & & \\
\hline \multicolumn{5}{|l|}{ CINGULATA } \\
\hline \multirow[t]{2}{*}{ Dasypodidae } & Dasypus novemcinctus Linnaeus, 1758 & Tatu-galinha & & \\
\hline & Euphractus sexcinctus (Linnaeus, 1758) & Tatu-peba & & \\
\hline \multicolumn{5}{|c|}{ DIDELPHIMORPHIA } \\
\hline \multirow[t]{4}{*}{ Didelphidae } & Didelphis aurita Wied-Neuwied, 1826 & Gambá-de-orelha-preta & & \\
\hline & $\begin{array}{l}\text { Metachirus nudicaudatus (É. Geoffroy, } \\
\text { 1803) }\end{array}$ & Cuíca-de-quatro-olhos & & \\
\hline & Monodelphis americana (Müller, 1776) & Cuíca-de-três-listras & & \\
\hline & Philander frenatus (Olfers, 1818) & Cuíca-de-quatro-olhos & & \\
\hline \multicolumn{5}{|l|}{ PRIMATES } \\
\hline Callitrichidae & Callithrix flaviceps (Thomas, 1903) & Sagui-da-Serra & EM & EM \\
\hline \multicolumn{5}{|l|}{ RODENTIA } \\
\hline Sciuridae & Guerlinguetus ingrami (Thomas, 1901) & Caxinguelê & & \\
\hline Cuniculidae & Cuniculus paca (Linnaeus, 1766) & Paca & & \\
\hline
\end{tabular}

Três táxons foram classificados como abundantes (CTA): Didelphis aurita, Nasua nasua e Canis lupus familiaris. Sete táxons se apresentaram como comuns: Monodelphis americana, Euphractus sexcintus, Dasypus novemcinctus, Callithrix flaviceps, Leopardus pardalis, Cuniculus paca e Mazama americana. Oito táxons se apresentaram como raros: Metachirus nudicaudatus, Philander frenatus, Puma yagouaroundi, Puma concolor, Eira barbara, Galictis cuja, Guerlinguetus ingrami e Mazama bororo (Tabela 2).

As espécies mais abundantes foram Nasua Nasua, com Abundância Relativa de 26,19\% e Canis lupus familiares com 15,48 \% (Tabela 2).

Requer atenção a ocorrência de espécies como Callithrix flaviceps, animal raro, mas sendo comum seu registro na área, espécie que se encontra Em Perigo (EM) (ICMBIO, 2018), bem como os felinos Leopardus pardalis e Puma concolor, cujas presenças são diagnósticas da relevância da área.

Leopardus pardalis consta como Menos Preocupante (LC) pelo ICMBIO (2018) devido à sua ampla distribuição, contudo segundo Caso et al. (2008), sua população está em declínio, aparecendo em situação Vulnerável (VU) (IEMA, 2005), sendo um animal difícil de ser encontrado e, normalmente pouco frequente. Apesar disso, a espécie teve o registro de 3 indivíduos durante o período de pesquisa.

Puma concolor aparece como Em Perigo (EN) para ambas as listas. Caso et al. (2008) discutem a 
situação da espécie, cuja conservação é considerada um desafio, por ser um predador de grande porte e, frente ao fato de já ter sido eliminada de grandes áreas de sua distribuição original. A caça e a alteração do habitat, com consequente redução da disponibilidade de presas, são as principais ameaças à sobrevivência da espécie (MICHALSKI, 2006; MICHALSKI et al., 2007). A supressão de mamíferos de grande porte, como o P. concolor, se faz preocupante devido à perda das funcionalidades ecológicas essenciais que eles exercem (CUARÓN, 2000).

Tabela 2: Relação do número de espécies (N), Abundância Relativa (AR), Abundância (A), Classe de Abundância (CTA), Frequência de Ocorrência (FO) e Classe de Frequência (CF), registradas no Sítio Morro Branco, Vargem Alta, ES. Abundância $(A)=$ PA: Pouco Abundante; A: Abundante; MA: Muito Abundante. Classe de Abundância $(C T A)=A$ : Abundante; C: Comum; R: Raro. Classe de Frequência= MF: Muito Frequente; F: Frequente; PF: Pouco Frequente.

\begin{tabular}{|c|c|c|c|c|c|c|}
\hline Ordem / Espécie & $\mathbf{N}$ & AR (\%) & A & CTA & FO (\%) & $\mathrm{CF}$ \\
\hline \multicolumn{7}{|l|}{ ARTIODACTYLA } \\
\hline Mazama americana (Erxleben, 1777) & 3 & 3,57 & A & $\mathrm{C}$ & 12,5 & $\mathrm{PF}$ \\
\hline Mazama bororo Duarte, 1996 & 1 & 1,19 & PA & $\mathrm{R}$ & 4,17 & PF \\
\hline \multicolumn{7}{|l|}{ CARNIVORA } \\
\hline Canis lupus familiaris (Linnaeus, 1758) & 13 & 15,48 & $\mathrm{MA}$ & A & 33,33 & $\mathrm{~F}$ \\
\hline Leopardus pardalis (Linnaeus, 1758) & 3 & 3,57 & A & $\mathrm{C}$ & 12,5 & $\mathrm{PF}$ \\
\hline Puma concolor (Linnaeus, 1771) & 1 & 1,19 & PA & $\mathrm{R}$ & 4,16 & $\mathrm{PF}$ \\
\hline Puma yagouaroundi (É. Geoffroy, 1803) & 1 & 1,19 & PA & $\mathrm{R}$ & 4,16 & PF \\
\hline Eira barbara (Linnaeus, 1758) & 1 & 1,19 & PA & $\mathrm{R}$ & 4,16 & PF \\
\hline Galictis cuja (Molina, 1782) & 1 & 1,19 & $\mathrm{PA}$ & $\mathrm{R}$ & 4,16 & $\mathrm{PF}$ \\
\hline Nasua nasua (Linnaeus, 1766) & 22 & 26,19 & $\mathrm{MA}$ & A & 12,5 & PF \\
\hline \multicolumn{7}{|l|}{ CINGULATA } \\
\hline Dasypus novemcinctus (Linnaeus, 1758) & 6 & 7,14 & $\mathrm{MA}$ & $\mathrm{C}$ & 25 & $\mathrm{~F}$ \\
\hline Euphractus sexcinctus (Linnaeus, 1758) & 4 & 4,76 & A & $\mathrm{C}$ & 16,66 & $\mathrm{PF}$ \\
\hline \multicolumn{7}{|l|}{ DIDELPHIMORPHIA } \\
\hline Didelphis aurita Wied-Neuwied, 1826 & 12 & 14,29 & $\mathrm{MA}$ & A & 50 & $\mathrm{MF}$ \\
\hline Metachirus nudicaudatus (É. Geoffroy, 1803) & 1 & 1,19 & PA & $\mathrm{R}$ & 4,16 & $\mathrm{PF}$ \\
\hline Monodelphis americana (Müller, 1776) & 4 & 4,76 & $\mathrm{~A}$ & $\mathrm{C}$ & 16,66 & $\mathrm{PF}$ \\
\hline Philander frenatus (Olfers, 1818) & 2 & 2,38 & $\mathrm{PA}$ & $\mathrm{R}$ & 8,33 & $\mathrm{PF}$ \\
\hline \multicolumn{7}{|l|}{ PRIMATES } \\
\hline Callithrix flaviceps (Thomas, 1903) & 4 & 4,76 & A & $\mathrm{C}$ & 4,16 & $\mathrm{PF}$ \\
\hline \multicolumn{7}{|l|}{ RODENTIA } \\
\hline Cuniculus paca (Linnaeus, 1766) & 3 & 3,57 & $\mathrm{~A}$ & $\mathrm{C}$ & 8,33 & $\mathrm{PF}$ \\
\hline Guerlinguetus ingrami (Thomas, 1901) & 2 & 2,38 & PA & $\mathrm{R}$ & 8,33 & PF \\
\hline
\end{tabular}

$\mathrm{AR}=\geq 5 \mathrm{MA}$ (muito abundante; $<5$ e $>2,4 \mathrm{~A}$ (abundante) $<<2,5 \mathrm{PA}$ (pouco abundante).

$\mathrm{FO}=\geq 50 \mathrm{MF}$ (muito frequente); $<50$ e $\geq 25 \mathrm{~F}$ (frequente) $<25 \mathrm{PF}$ (pouco frequente).

Em relação ao número de espécies $(n=18)$, a área de estudo, mesmo considerada como um fragmento que durante muito tempo sofreu e ainda sofre fortes interferências antrópicas e hoje encontrase em estágio secundário de regeneração, apresenta resultados compatíveis alcançados por outros estudos com mamíferos não-voadores na região sul Capixaba. Vale et al. (2015) registraram 15 espécies em um estudo realizado no Parque Estadual da Cachoeira da Fumaça. Oliveira et al. (2020) registraram 17 espécies para a Reserva Particular do Patrimônio Natural (RPPN) Mata do Macuco. Para a Floresta Nacional (FLONA) de Pacotuba, foram registradas 20 espécies no último plano de manejo (ICMBIO, 2011).

Pôde-se observar uma leve tendência à estabilização da curva de amostragem (Figura 2), sendo necessário um prolongamento dos esforços de amostragem. A riqueza de espécies observada $(S=18)$ ficou aquém do esperado pelo Teste de Jacknife $1(23,93)$, uma diferença de 5 espécies. Observa-se que poderiam ter sido registradas, no mínimo, mais 2 espécies $(S d)$ e, no máximo, mais $10(J+S d)$, caso houvessem sido amostrados 100 indivíduos. Esta diferença pode ter sido causada pela perturbação 
provocada pela presença de cães na área. A Eficiência de Coleta (EC) foi de aproximadamente 75\% (Tabela3).

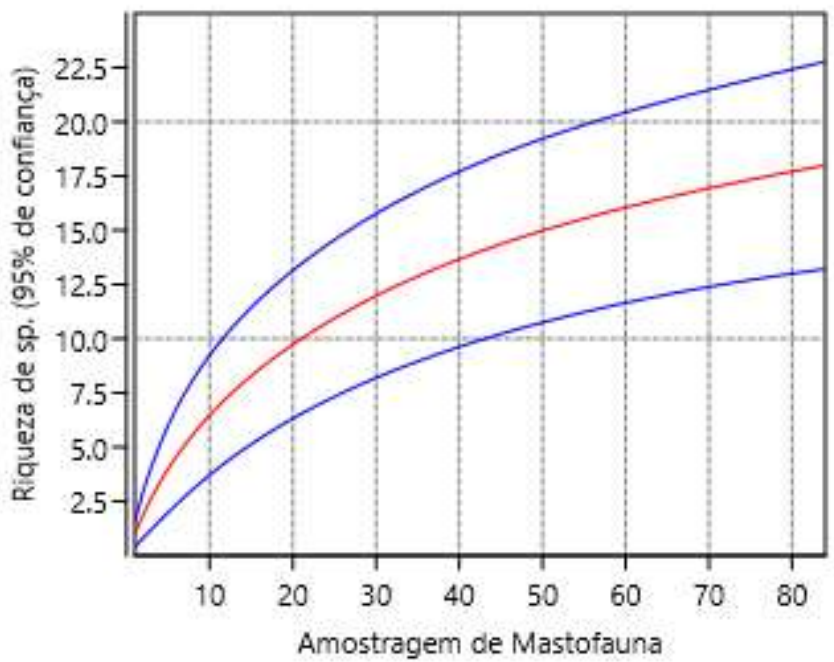

Figura 2: Curva de rarefação para riqueza de espécies do Sítio Morro Branco, Vargem Alta, ES.

Tabela 3: Estimadores de riqueza das espécies de mamíferos não-voadores do Sítio Morro Branco, Vargem Alta, ES.

\begin{tabular}{|l|l|l|l|l|l|l|}
\hline S & Shannon_H & Jacknife & Sd & J+Sd & J-Sd & EC \\
\hline 18 & 2,393 & 23,93 & 2,35 & 28,28 & 21,58 & $75,21 \%$ \\
\hline
\end{tabular}

Uma comparação importante deve ser feita com o estudo de Fiorese et al. (2020), realizado na RPPN Mata da Serra, que está localizada muito próximo à região de Morro Branco, sendo distantes em aproximadamente $8 \mathrm{~km}$, separadas por um mosaico de fragmentos de vegetação nativa em diferentes estágios de regeneração e plantios de eucaliptos. Na RPPN Mata da Serra foram registradas 15 espécies e Índice de Shannon de 2,520, pouco maior que o registrado para a região de Morro Branco, que foi de 2,393 (Tabela 3).

Conforme Tabela 4, para a condução deste estudo foram utilizadas diferentes técnicas: registro visual, armadilha fotográfica, armadilha de gaiolas, procura por vestígios (pegadas e fezes) e registros de indivíduos mortos por cães. A combinação dessas técnicas tem sido tem utilizadas em outros estudos (VOSS et al., 1996; PASSAMANI et al., 2000; PEREIRA et al., 2001; RABELLO et al., 2009).

Destaca-se o baixo número de espécies registrado pelo emprego das armadilhas de gaiola, com apenas duas espécies de Didelphidae, sendo Metachirus nudicaudatus registrado apenas por esse método. Esse fato pode ser justificado pelo hábito da espécie, que utiliza o solo para se locomover de forma predominante (MILES et al., 1981; FLECK et al., 1995; CUNHA et al., 2002; GRELLE, 2003; SANTOS et al., 2004; ASTÚA et al., 2006). É um animal noturno e solitário, sendo muito arisco, dado a esse fato, é difícil de ser encontrado, pois fogem ao menor ruído.

A técnica que registrou maior número de táxons foi o registro de carcaças de animais predados, com 9 espécies, seguido de registro visual, com 8 espécies, e armadilha fotográfica com 7 espécies (Tabela 4).

O emprego do registro de carcaças de animais predados por cães (C. lupus familiaris) na área não se mostra relevante apenas pelo alto número de espécies que abrangeu, como também porque foi o método com maior número de registros exclusivos, com $P$. yagouaroundi, G. cuja e $M$. bororo registrados apenas 
desta forma.

Ao todo, são 13 animais encontrados abatidos, correspondendo a $18 \%$ do total de indivíduos registrados (Tabela 4). Vale ressaltar que $C$. lupus familiaris obteve a segunda maior representatividade na área, com Abundância Relativa de 15,48 \%. Os cães são considerados os carnívoros mais abundantes em várias áreas naturais (HUGHES et al., 2013), incluindo a Mata Atlântica (PASCHOAL et al., 2012), ocorrendo em números muito mais altos que os carnívoros nativos, geralmente presentes em baixas densidades, como o P. concolor e P. yagouaroundi, ambos pouco abundantes na área. Mesmo L. pardalis, que foi classificado como abundante (Tabela 2), apresenta apenas 3,57 \% de Abundância Relativa.

A espécie mais predada foi $D$. aurita, com 3 ocorrências, seguida de $D$. novemcinctus e $M$. americana, ambas com dois indivíduos; M. americana, E. sexcintus, P. yagouaroundi, G. cuja, N. nasua e M. bororo, com um indivíduo cada. No estudo de Rangel et al. (2013), acerca da predação de vertebrados por Canis lupus familiaris no Jardim Botânico do Rio de Janeiro, a espécie mais atingida também foi $D$. aurita, em 86,11\% dos casos.

Tabela 4: Tipo de registro realizado para cada espécie e relação de número de indivíduos vivos e encontrados mortos por ataques de cães no Sítio Morro Branco, Vargem Alta, ES. 1= Registro Visual; 2= Armadilha de Gaiola; 3= Armadilha Fotográfica; $4=$ Vestígios; $5=$ Carcaça.

\begin{tabular}{|c|c|c|c|}
\hline Ordem / Espécie & Tipo de registro & Contagem indivíduos vivos & Contagem indivíduos mortos \\
\hline \multicolumn{4}{|l|}{ ARTIODACTYLA } \\
\hline Mazama americana (Erxleben, 1777) & $1 ; 5$ & 1 & 2 \\
\hline Mazama bororo Duarte, 1996 & 5 & 0 & 1 \\
\hline \multicolumn{4}{|l|}{ CARNIVORA } \\
\hline Canis lupus familiaris (Linnaeus, 1758) & $3 ; 4$ & 13 & 0 \\
\hline Leopardus pardalis (Linnaeus, 1758) & $1 ; 4$ & 3 & 0 \\
\hline Puma concolor (Linnaeus, 1771) & 4 & 1 & 0 \\
\hline Puma yagouaroundi (É. Geoffroy, 1803) & 5 & 0 & 1 \\
\hline Eira barbara (Linnaeus, 1758) & 4 & 1 & 0 \\
\hline Galictis cuja (Molina, 1782) & 5 & 0 & 1 \\
\hline Nasua nasua (Linnaeus, 1766) & $3 ; 4 ; 5$ & 21 & 1 \\
\hline \multicolumn{4}{|l|}{ CINGULATA } \\
\hline Dasypus novemcinctus (Linnaeus, 1758) & $1 ; 3 ; 5$ & 4 & 2 \\
\hline Euphractus sexcinctus (Linnaeus, 1758) & $1 ; 5$ & 3 & 1 \\
\hline \multicolumn{4}{|l|}{ DIDELPHIMORPHIA } \\
\hline Didelphis aurita Wied-Neuwied, 1826 & $1 ; 3 ; 5$ & 9 & 3 \\
\hline Metachirus nudicaudatus (É. Geoffroy, 1803) & 2 & 1 & 0 \\
\hline Monodelphis americana (Müller, 1776) & $1 ; 3 ; 5$ & 3 & 1 \\
\hline Philander frenatus (Olfers, 1818) & $2 ; 3$ & 2 & 0 \\
\hline \multicolumn{4}{|l|}{ PRIMATES } \\
\hline Callithrix flaviceps (Thomas, 1903) & 1 & 4 & 0 \\
\hline \multicolumn{4}{|l|}{ RODENTIA } \\
\hline Cuniculus paca (Linnaeus, 1766) & 3 & 3 & 0 \\
\hline Guerlinguetus ingrami (Thomas, 1901) & 1 & 2 & 0 \\
\hline Total & - & 71 & 13 \\
\hline
\end{tabular}

A maior parte destas espécies aparece com frequência em listas de espécies predadas ou vítimas de interações ecológicas negativas com C. lupus familiaris. No estudo de Pereira et al. (2019), sobre mamíferos silvestres predados por cães domésticos em fragmentos de Mata Atlântica, nove das espécies registradas ocorrem também na região de Morro Branco: $D$. aurita, $M$. nudicaudatus, $P$. frenatus, $D$. novemcinctus, $E$. sexcintus, E. barbara, G. cuja, N. nasua e C. paca. Todas essas espécies também foram predadas na área de estudo, com exceção de M. nudicaudatus. 
No estudo de Lessa et al. (2016), sobre espécies predadas por cães em áreas protegidas no Brasil, constam as espécies $D$. aurita, $D$. novemcintus, E. sexcintus, N. nasua e E. barbara. O felino $P$. yagouaroundi também é citado no mesmo estudo, embora referido como vítima de competição e não de predação, como o ocorrido em Morro Branco. Cabe destacar que, em Morro Branco, o indivíduo de P. yagouaroundi, registrado através de carcaça, foi o único representante da espécie neste levantamento. Deve-se dar atenção ao ataque de cães a esta espécie, pois naturalmente sua população ocorre com baixa densidade (ALMEIDA et al., 2013), representando um risco para a sobrevivência da espécie.

Os cães geralmente não caçam verdadeiramente, pois a predação é definida como o ato de capturar (direta ou indiretamente) e se alimentar da presa (STRAUSS, 1991). Ao invés disso, geralmente perseguem e capturam outras espécies para se divertir. Nestes jogos de predadores - presas eles podem ferir animais, levando-os à morte, embora nem sempre se alimentem deles (GOMPPER, 2015). Além disso, pode haver competição com predadores nativos (BRICKNER, 2003; CAMPOS et al., 2007; OLIVEIRA et al., 2008; HUGHES et al., 2013).
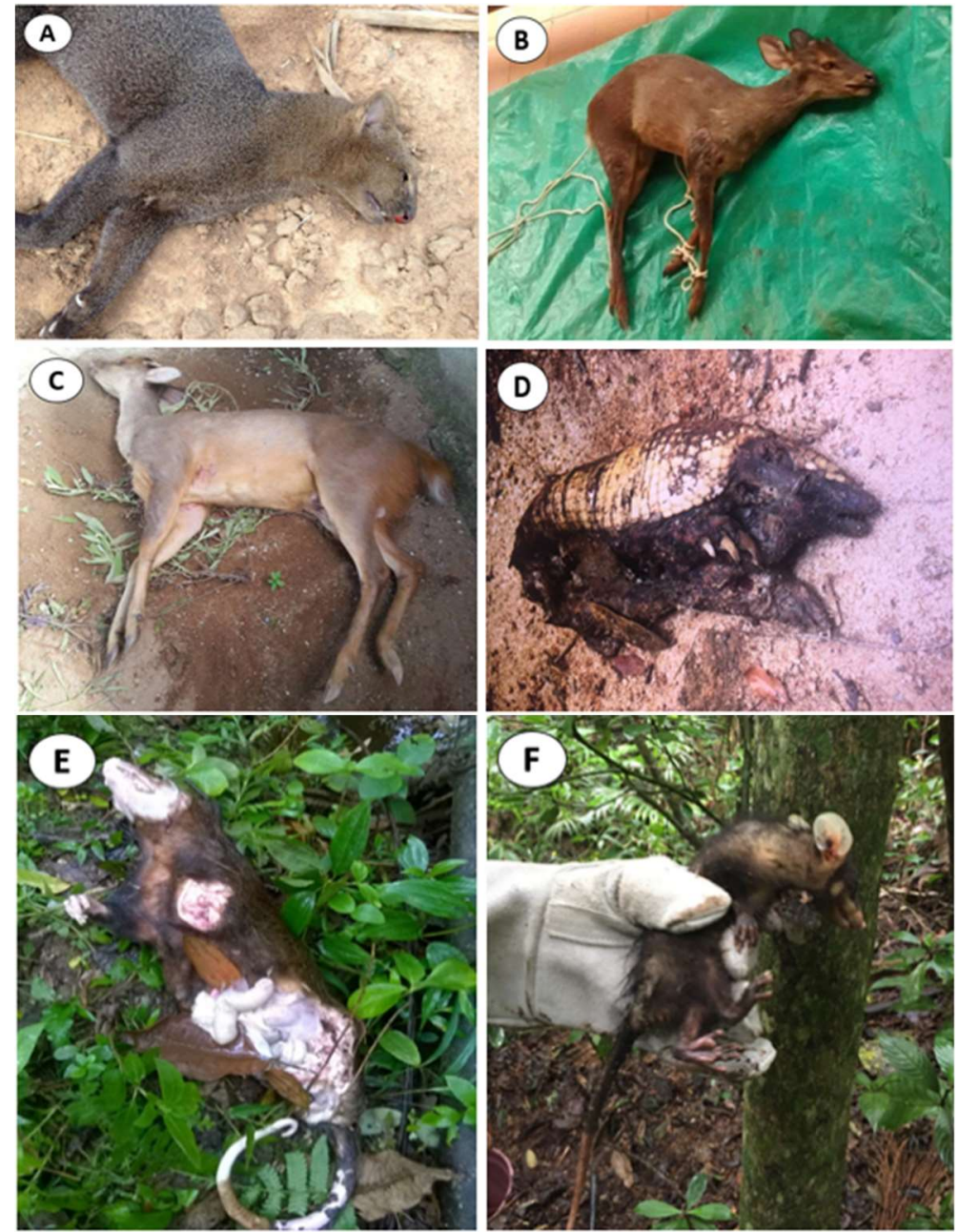

Figura 3: Carcaças dos animais abatidos por cães domésticos. A: Puma yagouaroundi; B: Mazama bororo; C: Mazama americana; D: Euphractus sexcintus; E e F: Didelphis aurita.

A característica de $C$. lupus familiaris de matar, mas não se alimentar de suas vítimas pode ser observada na Figura 3, carcaças de animais que foram abatidos, com diversas marcas de mordida e lutas, sem o consumo da carne. 
O estudo de Rangel et al. (2013), acerca do ataque à vertebrados por C. lupus familiaris no Jardim Botânico do Rio de Janeiro, os mamíferos se destacaram como $80 \%$ das espécies atacadas por cães, e $94 \%$ do total de animais vitimados, quase todos eles inteiros, sem nenhuma parte consumida.

Alguns estudos apontam para uma alta proporção de cães em relação às espécies nativas dentro de remanescentes florestais, além de eventos de predação e competição, alertando sobre a necessidade de considerar o cão doméstico como importante fator de deterioração da biodiversidade (SRBEK-ARAUJO et al., 2008; ESPARTOSA, 2009). Esses dados corroboram com o encontrado na área de estudo, onde $C$. lupus familiaris apresentou a segunda maior Abundância Relativa entre as espécies (15,48 \%).

Para a região sul-capixaba, são escassas e incompletas as informações desta natureza, sendo que a maior parte dos estudos direcionados para a mastofauna não possuem, entre seus objetivos, o registro de $C$. lupus familiaris e seu impacto sobre os mamíferos nativos. O estudo feito por Vale et al. (2015), no Parque Estadual Cachoeira da Fumaça, não cita registro de C. lupus familiaris. Já no estudo de Oliveira et al. (2020), na Mata da Macuco, houve registro, porém, como a espécie menos abundante na área. Na FLONA de Pacotuba (BRASIL, 2018), há registro de visitas frequentes de cães na área da UC, através de armadilhas fotográficas, em posição ou situação de ataque a animais silvestres, embora não haja contagem de indivíduos de $C$. Iupus familiaris, nem registro de carcaças.

Pereira et al. (2019), realizando um levantamento abrangendo trabalhos publicados com registros de predação por C. lupus familiaris no Brasil, chegou a um resultado de 27 espécies predadas, sendo alta a proporção de cães em remanescentes florestais, mais recorrentes em regiões de Mata Atlântica, incluindo áreas de proteção, como as UCs. Altas densidades de cães em áreas naturais podem, a princípio, afetar carnívoros nativos devido à competição (LESSA et al., 2016), como é o caso de Felidae, Mustelidae, Procyonidae e Canidae, famílias da ordem Carnívora encontradas em Vargem Alta.

A maior parte dos registros compilados por Pereira et al. (2019) ocorreu em regiões de transição entre espaço rural e o urbano. Diferentemente do campo, nestas áreas existe uma densidade populacional maior de C. lupus familiaris, podendo até mesmo exceder o número de pessoas (HUGHES et al., 2013). Este cenário transicional é identificado na região de Morro Branco, distante poucos quilômetros do centro do município de Vargem Alta e à beira de uma movimentada rodovia estadual.

Uma medida para contornar tal cenário impactante de espécie exótica invasora, seria o controle populacional, evitando assim a invasão de ambientes naturais por esta espécie (ESPARTOSA, 2009). No Brasil, desde 2018 foi regulamentada a Resolução №7 do Ministério do Meio Ambiente, chamada de "Estratégia Nacional para Espécies Exóticas Invasoras" (BRASIL, 2018). Contudo, ainda assim o país carece de medidas legais, como um plano de manejo de cães, que desenvolva projetos de controle populacional desses animais e uma legislação assertiva para conservação das espécies nativas (GARCIA et al., 2012).

Dessa forma, a região do presente estudo carece de um tratamento conservacionista visando os mamíferos com ocorrência na área, visto que além de algumas espécies estarem ameaçadas de extinção, também estão sob ameaça de ataques por cães. O papel ecológico dos mamíferos é notório, interagindo com várias outras espécies, sendo importantes para o estabelecimento tanto de espécies animais, quanto 
vegetais e, conforme Penter et al. (2008), essas interações ecológicas são importantes principalmente em ambientes fragmentados.

\section{CONCLUSÕES}

Em comparação com estudos efetuados em áreas de mesma tipologia vegetacional e extensão semelhante, como a RPPN Mata da Serra no mesmo município, o sítio Morro Branco apresenta considerável diversidade de mamíferos, com uma composição faunística ainda pouco estudada, incluindo a ocorrência de espécies raras e ameaçadas.

Evidencia-se o impacto significativo de Canis lupus familiaris sobre a mastofauna, suprimindo espécies nativas e representando um risco para a conservação de suas populações locais. Desta forma, tornase importante o fomento a ações direcionadas à redução ou erradicação do impacto de cães domésticos em trechos florestais fragmentados e vulneráveis.

Ressalta-se a importância ecológica da área de estudo, bem como a necessidade de inclusão de medidas específicas contra o impacto de Canis lupus familiaris na elaboração do futuro plano de manejo para a criação da Área de Proteção Ambiental (APA) "Morro Branco". Dado o exposto, este estudo contribui para agregar, não somente ao conhecimento sobre a mastofauna da região sul-capixaba, mas também acerca do impacto de cães domésticos sobre a fauna silvestre, visto que são poucos os estudos existentes sobre a mastofauna no estado do Espírito Santo e, mais escassos ainda, aqueles que registram o impacto causado por cães a este grupo de fauna.

\section{REFERÊNCIAS}

AHUMADA, J. A.; SILVA, C. E.; GAJAPERSAD, K.; HALLAM, C.; HURTADO, J.; MARTIN, E.; ANDELMAN, S. J.. Community structure and diversity of tropical forest mammals: data from a global camera trap network. Philosophical Transactions of the Royal Society B: Biological Sciences, v.366, n.1578, p.2703-2711, 2011. DOI: https://doi.org/10.1098/rstb.2011.0115

ALMEIDA, L. B.; QUEIROLO, D.; BEISIEGEL, B. M.; OLIVEIRA, T. G.. Avaliação do estado de conservação do Gato-mourisco Puma yagouaroundi (É. Geoffroy Saint-Hilaire, 1803) no Brasil. Biodiversidade Brasileira, v.3, n.1, p.99-106, 2013.

ASTÚA, D.; MOURA, R. T.; GRELLE, C. E. V.; FONSECA, M. T.. Influence of baits, trap type and position for small mammals capture in a Brazilian lowland Atlantica Forest. Boletim do Museu de Biologia Mello Leitão, v.19, p.31-44, 2006.

BAILLIE, J. E. M.; HILTON-TAYLOR, C.; STUART, S. N.. 2004 IUCN red list of threatened species: a global species assessment. Cambridge: IUCN, 2004.

BECKER, M.; DALPONTE, J. C.. Rastros de mamíferos silvestres brasileiros: um guia de campo. 2 ed. Brasília: Universidade de Brasília, 1991.

BIERREGAARD, R. O.; LOVEJOY, T. E.; KAPOS, V.; SANTOS, A. A. S.; HUTCHINGS, R. W.. The biological dynamics of tropical rainforest fragments: A prospective comparison of fragment and continuous forest. BioScience, v.42, n.11, p.859-866, 1992.

BRASIL. Resolução $\mathbf{n . 7}$ de $\mathbf{2 7}$ de maio de 2018. Estratégia Nacional para Espécies Exóticas Invasoras. Brasília: Ministério do Meio Ambiente, 2018.

BRICKNER, I.. The Impact of Domestic dogs (Canis familiaris) on Wildlife Welfare and Conservation: A Literature Review with a Situation Summary from Israel. Internal Report, Israel Park and Nature Authority. Tel Aviv: Department of Zoology, University, 2003.

BROOKS, T.; MITTERMEIER, R. A.; MITTERMEIER, C. G.; FONSECA, G. A. B.; RYLANDS, A. B.; KONSTANT, W. R.; FLICK, P.; PILGRIM, J.; OLDFIELD, S. F.; MAGIN, G.; HILTON-TAYLOR, C.. Habitat Loss and Extinction in the Hotspots of Biodiversity. Conservation Biology, v.16, p.909-923, 2002. DOI: https://doi.org/10.1046/j.1523-1739.2002.00530.x

CAMPOS, C. B.; ESTEVES, C. F.; FERRAZ, K. M. P. M. B.; CRAWSHAW JUNIOR, P. G.; VERDADE, L. M.. Diet of freeranging cats and dogs in a suburban and rural environment, south-eastern Brazil. Journal of Zoology, v.273, n.1, p.14-20, 2007.

CASO, A.; LOPEZ-GONZALEZ, C.; PAYAN, E.; EIZIRIK, E.; OLIVEIRA, T.; LEITE-PITMAN, R.; KELLY, M.; VALDERRAMA, C.; LUCHERINI, M.. Puma concolor. IUCN Red List of Threatened 
Species, 2008.

CEBALLOS, G.; EHRLICH, P.. Global Biodiversity Hotspots and Conservation: Insights from Mammal Distributions. Proceedings of the National Academy of Sciences of the United States of America (PNAS), v.103, n.51, p.1937419379, 2006. DOI: https://doi.org/10.1073pnas.0609334103

CHIARELLO, A. G.. Effects of fragmentation of the Atlantic forest on mammal communities in south-eastern Brazil. Biological Conservation, v.89, p.71-82, 1999. DOI: https://doi.org/10.1016/S0006-3207(98)00130-X

CUARÓN, A. D.. A global perspective on habitat disturbance and tropical rainforest mammals. Conservation Biology, v.14, n.6, p.1574-1579, 2000.

CUNHA, A. A.; VIEIRA, M. V.. Support diameter, incline, and vertical movements of four didelphid marsupials in the Atlantic forest of Brazil. Journal of Zoology, v.258, p.419426, 2002. DOI: https://doi.org/10.1017/S0952836902001565

ESPARTOSA, K. D.. Mamíferos terrestres de maior porte e a invasão de cães domésticos em remanescentes de uma paisagem fragmentada de Mata Atlântica: avaliação da eficiência de métodos de amostragem e da importância de múltiplos fatores sobre a distribuição das espécies. Dissertação (Mestrado em Ecologia: Ecossistemas Terrestres e Aquáticos) - Universidade de São Paulo, São Paulo, 2009. DOI: http://doi.org/10.11606/D.41.2009.tde-01052009$\underline{125425}$

FERNANDEZ, F. A. S.. Efeitos da fragmentação de ecossistemas: a situação das Unidades de Conservação. In: CONGRESSO BRASILEIRO DE UNIDADES DE CONSERVAÇÃO. Anais. Curitiba: Rede Nacional Pró-Unidades de Conservação, 1997. p.48-68.

FIORESE, C. H. U.; BISSA, L. G.; RABELLO, H.; SILVA FILHO, G.; BINDELI, G. M.. Inventário de mamíferos não voadores da reserva particular do patrimônio natural Mata da Serra (ES). Nature and Conservation, v.13, n.2, p.1-13, 2020. DOI: http://doi.org/10.6008/CBPC23182881.2020.002.0001

FLECK, D. W.; HARDER, J. D.. Ecology of marsupials in two Amazonian Rain Forests in Northeastern Peru. Journal of Mammalogy, v.76, p.809-818, 1995.

GALETTI, M.; SAZIMA, I.. Impacto de cães ferais em um fragmento urbano de Floresta Atlântica no sudeste do Brasil. Natureza \& Conservação, v.4, n.1, p.58-63, 2006.

GALETTI, M.; GIACOMINI, H.; SILVEIRA, R. B.; BERNARDO, C.; MARQUES, R.; BOVENDORP, R.; STEFFLER, C.; RUBIM, P.; GOBBO, S.; DONATTI, C.; ANZOLIN, R. B.; MEIRELLES, F.. Priority areas for the conservation of Atlantic forest large mammals. Biological Conservation, v.142, p.1229-1241, 2009. DOI: http://doi.org/10.1016/j.biocon.2009.01.023

GALETTI, M.; GUEVARA, R.; NEVES, C. L.; RODARTE, R. R.; BOVENDORP, R. S.; MOREIRA, M.; HOPKINS, J. B.; YEAKEL, J. D.. Defaunation affects the populations and diets of rodents in Neotropical rainforests. Biological Conservation, v.190, p.2-7, 2015

GARCIA, R. C. M.; CALDERÓN, N.; FERREIRA, F.. Consolidação de diretrizes internacionais de manejo de populações caninas em áreas urbanas e proposta de indicações para seu gerenciamento. Revista Panamericana de Salud Publica, v.32, n.2, p.140-144, 2012.

GREEN, J. S.; GIPSON, P. S.. Feral dogs. The Handbook: Prevention and Control of Wildlife Damage, 1994.

GRELLE, C. E. V.. Forest structure and vertical stratification of small mammals in a secondary Atlantic Forest, Southeastern Brazil. Studies on Neotropical Fauna and Environment, v.38, p.81-85, 2003.

GOMPPER, M. E.. Free-ranging dogs and wildlife conservation. Oxford Scholarship Online, 2015. DOI: http://dx.doi.org/10.1093/acprof:osobl/9780199663217.001 .0001

HELDER-JOSÉ; ZORTEA, M.; PASSAMANI, J. A.; MENDES, S. L.; PASSAMANI, M.. Mammals from Duas Bocas Biological Reserve, state of Espírito Santo, Brazil. Bol. Mus. Biol. Mello Leitão, v.38, n.2, p.163-180, 2016.

HUGHES, J.; MACDONALD, D. W.. A review of the interactions between free-roaming domestic dogs and wildlife. Biological Conservation, Boston, v.157, p.341-351, 2013. DOI: https://doi.org/10.1016/j.biocon.2012.07.005

ICMBIO. Instituto Chico Mendes de conservação da biodiversidade. Plano de manejo da Floresta Nacional de Pacotuba, localizada no estado do Espírito Santo. Brasília: ICMBIO, 2011.

ICMBIO. Instituto Chico Mendes de conservação da biodiversidade. Livro Vermelho da Fauna Brasileira Ameaçada de Extinção. Brasília: ICMBIO, 2018.

IEMA. Instituto de Meio Ambiente e Recursos Hídricos. Lista de espécies ameaçadas de extinção no Espírito Santo. Fauna ameaçada de extinção. Cariacica: IEMA, 2005.

IPEMA. Instituto de Permacultura e Ecovilas da Mata Atlântica. Áreas e ações prioritárias para a conservação da biodiversidade da Mata Atlântica no estado do Espírito Santo. Vitória: Instituto de Pesquisas da Mata Atlântica, 2011.

IWANAGA, S.. Levantamento de mamíferos diurnos de médio e grande porte no Parque Nacional do Jaú: resultados preliminares. In: BORGES, S. H.; IWANAGA, S.; DURIGAN, C. C.; PINHEIRO, M. R.. Janelas para a Biodiversidade no Parque Nacional do Jaú: uma estratégia para o estudo da biodiversidade na Amazônia. Manaus: Fundação Vitória Amazônica, 2004. p.195-207

LESSA, I.; GUIMARAES, T. C. S.; BERGALLO, H. G.; CUNHA, A. VIEIRA, E. M.. Domestic dogs in protected areas: a threat to Brazilian mammals? Natureza \& Conservação, v.14, n.2, p.46-56, 2016. DOI: http://dx.doi.org/10.1016/j.ncon.2016.05.001

MENDES, S. L.. Importância dos remanescentes de Mata Atlântica no Estado do Espírito Santo para a conservação de primatas. Cadernos de Pesquisa da UFES, v.4, p.1-14, 1995.

MENEZES, J. F. S.; FERNANDEZ, F. A. S.. Nestedness in forest mammals is dependent on area but not on matrix type and 
sample size: an analysis on different fragmented landscapes.

Braz. J. Biol., São Carlos, v.73, n.3, p.465-470, 2013. DOI: https://doi.org/10.1590/S151969842013000300002

\section{MICHALSKI, F.. Ecological consequences of habitat} fragmentation and disturbance in Amazonian forests. Thesis (Doctor of Philosophy) - University of East Anglia, Norwich, 2006.

MICHALSKI, F.; PERES, C. A.. Disturbance-mediated mammal persistence and abundance-area relationships in Amazonian forest fragments. Conserv Biol., v.21, n.6, p.1626-1640, 2007. DOI: https://doi.org/10.1111/j.15231739.2007.00797.x

MILES, M. A.; SOUZA, A. A.; POVOA, M. M.. Mammal tracking and nest location in Brazilian forest with an improved spooland-line device. Jornal of Zooogy, v.195, p.331-347, 1981.

MOREIRA, D. O.; COUTINHO, B. R.; MENDES, S. L.. O status do conhecimento sobre a fauna de mamíferos do Espírito Santo baseado em registros de museus e literatura científica. Biota Neotrop., v.8, n.2, 2008

OLIVEIRA, V. B.; LINARES, A. M.; CORRÊA, G. L. C.; CHIARELLO, A. G.. Predation on the black capuchin monkey Cebus nigritus (Primates: Cebidae) by domestic dogs Canis lupus familiaris (Carnívora: Canidae), in the Parque Estadual Serra do Brigadeiro, Minas Gerais, Brazil. Revista Brasileira de Zoologia, v.25, n.2, p.376-378, 2008. DOI: https://doi.org/10.1590/S0101-81752008000200026

OLIVEIRA, F.; ROSA, C.; TEIXEIRA, V.; RABELLO, H.; FIORESE, C; SILVA FILHO, G.; TEIXEIRA, C.; BINDELI, G.. Inventário de mamíferos não voadores na Reserva Particular do Patrimônio Natural Mata do Macuco, no município de Presidente Kennedy, ES, Brasil. Revista Agraria Acadêmica, v.3, p.87-103, 2020. DOI: https://doi.org/10.32406/v3n32020/87-103/agrariacad

PAGLIA, A. P.; FONSECA, G. A. B.; RYLANDS, A. B.; HERRMAN, G.; AGUIAR, L. M. S.; CHIARELLO, A. G.; LEITE, Y. L. R.; COSTA, L. P.; SICILIANO, S.; KIERULFF, M. C. M.; MENDES, S. L.; TAVARES, V. C.; MITTERMEIER, R. A.; PATTON, J. L.. Lista anotada dos mamíferos do Brasil. Occasional Papers in Conservation Biology 6. 2 ed. Washington: Conservation International, 2012.

PARDINI, R.; UMETSU, F.. Pequenos mamíferos nãovoadores da Reserva Florestal do Morro Grande: distribuição das espécies e da diversidade em uma área de Mata Atlântica. Biota Neotrop., Campinas, v.6, n.2, 2006. DOI: https://doi.org/10.1590/S1676-06032006000200007

PASSAMANI, M.; MENDES, S.; CHIARELLO, A.. Non-volant mammals of the Estação Biológica de Santa Lúcia and adjacent areas of Santa Teresa, Espírito Santo, Brazil. Boletim do Museu de Biologia Mello Leitão, v.11, p.201214, 2000

PASCHOAL, A.; MASSARA, R.; SANTOS, J.; CHIARELLO, A.. Is the domestic dog becoming an abundant species in the Atlantic Forest? A study case in southeastern Brazil.

Mammalia, v.76, p.67-76, 2012. DOI:

https://doi.org/10.1515/mammalia-2012-0501

PAZ, P. R.; VENTURINI, A.. Guia ilustrado de mamíferos da
Reserva Natural da Vale do Rio Doce. Vila Velha: Originalis Natura, 2003.

PENTER, C.; PEDÓ, E.; FABIÁN, M.; HARTZ, S.. Inventário rápido da fauna de mamíferos do Morro Santana, Porto Alegre, RS. Revista Brasileira de Biociências, v.6, p.117-125, 2008.

PEREIRA, L. G.; TORRES, S. E. M.; SILVA, H. S.; GEISE, L. Nonvolant mammals of ilha grande and adjacent areas in southern Rio de Janeiro state, Brazil. Rio de Janeiro: Boletim do Museu Nacional (zoologia), 2001.

PEREIRA, A. D.; ANTONIAZZI, M. H.; VIDOTTO-MAGNONI, A. P.; ORSI, M. L.. Mamíferos silvestres predados por cães domésticos em fragmentos de Mata Atlântica no sul do Brasil. Biotemas, Florianópolis, v.32, n.2, p.107-113, 2019. DOI: https://doi.org/10.5007/2175-7925.2019v32n2p107

RABELLO, H.; LACCHINE, P. S.; PINHEIRO, P. C.; MOULIN, E.; VERISSIMO, A.. Levantamento da mastofauna nas áreas de reserva legal da fazenda Brunoro Agro - avicola, situada em Venda Nova do Imigrante - Espírito Santo, Brasil. In: CONGRESSO DE ECOLOGIA DO BRASIL, 10. Anais. São Lourenço, 2009.

RANGEL, C. H.; NEIVA, C. H. M. B.. Predação de Vertebrados por Cães Canis lupus familiaris (Mammalia: Carnívora) no Jardim Botânico do Rio de Janeiro, RJ, Brasil. Biodiversidade Brasileira, v.3, n.2, p.261-269, 2013. DOI: https://doi.org/10.37002/issn.2236-2886

REIS, N. R.; PERACCHI, A. L.; PEDRO, W. A.; LIMA, I. P.. Mamíferos do Brasil. 2 ed. Londrina, 2011.

RIBEIRO, M. C.; METZGER, J. P. W.; MARTENSEN, A. C.; PONZONI, F. J.; HIROTA, M. M.. The Brazilian Atlantic forest: how much is left, and how is the remaining forest distributed? Implications for conservation. Biological Conservation, Amsterdam, v.142, p.1141-1153, 2009. DOI: http://dx.doi.org/10.1016/j.biocon.2009.02.021

SANTOS, A. B.; LÓSS, S.; LEITE, Y. L. R.. Padrões de uso de estratos da floresta por pequenos mamíferos no Parque Estadual da Fonte Grande, Vitória, Espírito Santo. Natureza v.2, p.27-33, 2004

SRBEK-ARAUJO, A. C.; CHIARELLO, A. G.. Domestic dogs in Atlantic forest preserves of south-eastern Brazil: a cameratrapping study on patterns of entrance and site occupancy rates. Braz. J. Biol., São Carlos, v.68, n.4, p.771-779, 2008. DOI: https://doi.org/10.1590/S1519-69842008000400011

STRAUSS, S. Y.. Indirect effects in community ecology: Their definition, study and importance. Trends Ecol. Evol., v.6, n.7, p.206-210, 1991. DOI: https://doi.org/10.1016/01695347(91)90023-0

TABARELLI, M.; PINTO, L. P.; SILVA, J. M. C.; HIROTA, M. M.; BEDÊ, L. C.. Desafios e oportunidades para a conservação da biodiversidade na Mata Atlântica brasileira. Megadiversidade, v.1, p.132-138, 2005.

VALE, V.; PEREIRA, M. C. A.. Mamíferos do Parque Estadual Cachoeira da Fumaça. Natureza, v.13, n.5, p.234-239, 2015.

VEITCH, C. R.. Feral dog: a situation summary. Papakura: 
Endangered Species Recovery Council, 48 Manse Road, 2002.

\section{VOSS, R. S.; EMMONS, L. H.. Mammalian diversity in} neotropical lowland rainforests: a preliminary assessment. New York:

bulletin of the American museum of natural history, 1996.
YOUNG, J. K.; OLSON, K. A.; READING, R. P.;

AMGALANBAATAR, S.; BERGER, J.. Is wildlife going to the dogs? Impacts of feral and free-roaming dogs on wildlife populations. BioScience, v.61, p.125-132, 2011. DOI: https://doi.org/10.1525/bio.2011.61.2.7

A CBPC - Companhia Brasileira de Produção Científica (CNPJ: 11.221.422/0001-03) detém os direitos materiais desta publicação. Os direitos referem-se à publicação do trabalho em qualquer parte do mundo, incluindo os direitos às renovações, expansões e disseminações da contribuição, bem como outros direitos subsidiários. Todos os trabalhos publicados eletronicamente poderão posteriormente ser publicados em coletâneas impressas sob coordenação da Sustenere Publishing, da Companhia Brasileira de Produção Científica e seus parceiros autorizados. Os (as) autores (as) preservam os direitos autorais, mas não têm permissão para a publicação da contribuição em outro meio, impresso ou digital, em português ou em tradução. 